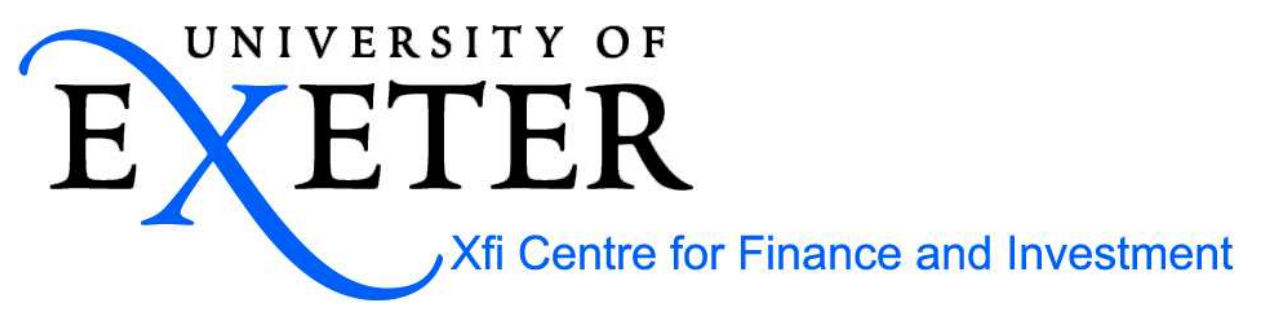

\title{
Retrieving Seasonally Adjusted Quarterly Growth Rates from Annual Growth Rates that are Reported Quarterly
}

\author{
Richard D.F. Harris \\ University of Exeter \\ Fatih Yilmaz \\ Bank of America
}

January 2006

Paper Number: 06/08

\begin{abstract}
Most macroeconomic time-series are reported on a quarterly basis. However, in many cases, official statistical agencies report annual (or 'year-on-year') growth rates rather than the quarterly data themselves. In this paper, we demonstrate how to recover seasonally adjusted quarterly growth rates from annual growth rates that are reported quarterly. As an illustration, we apply the technique to the highly seasonal UK Retail Sales series. Using only the annual growth rates calculated from the underlying data, we find that our procedure generates estimated seasonally adjusted quarterly growth rates that are almost indistinguishable from the 'true' seasonally adjusted quarterly growth rates.
\end{abstract}

Keywords: Seasonal adjustment; Annual growth; Year-on-year growth; Quarterly growth.

Address for correspondence: Professor Richard D. F. Harris, Xfi Centre for Finance and Investment, University of Exeter, Exeter EX4 4PU, UK. Tel: +44 (0) 1392 263215, Fax: +44 (0) 1392263242 Email: R.D.F.Harris@exeter.ac.uk. 


\section{Introduction}

Much empirical work in macroeconomics and finance makes use of quarterly observations on economic data, employing either the level of the variable or the quarterly growth rate. In most cases, the quarterly data are first seasonally adjusted to remove the cyclical component. For many economic series, however, official statistical agencies report annual (or 'year-on-year') growth rates rather than the underlying quarterly data themselves. This is particularly true for developing countries. For example, the Chinese Statistical Bureau reports quarterly year-on-year growth in CPI, but does not report the underlying quarterly CPI series. While annual growth rates remove some of the quarterly seasonality from the data, they yield a much smoother series than the true seasonally adjusted series because they are constructed using overlapping data. This lack of quarterly data presents a problem for empirical studies, and prevents the accurate estimation and testing of established economic theories for these countries. For example, for China, we are unable to calculate the quarterly real exchange rate and real interest rate and so we are unable to accurately test theories such as real interest parity using quarterly data. Moreover, the lack of quarterly data makes it difficult for market participants to accurately follow economic trends for these countries.

Since a given quarterly series of annual growth rates is consistent with an infinite number of possible series of quarterly growth rates, it is clearly not possible to retrieve the underlying (unadjusted) quarterly data from the observed annual growth rates. In particular, for a given economic series, we have $T$ unobserved quarterly growth rates, but only $T-3$ observed annual growth rates. Therefore, we would have to solve a system of $T-3$ equations in $T$ variables. In this paper, however, we show that while the original data themselves cannot be identified, we can retrieve the seasonally adjusted quarterly growth rates and from these, impute the seasonally adjusted series itself, in index form. Since empirical work in economics typically employs seasonally adjusted data, this should be sufficient for most studies that require quarterly observations on an economic series. As an illustration, we apply our technique to the highly seasonal UK Retail Sales series. In particular, we use annual growth rates calculated from the underlying quarterly data to estimate the corresponding seasonally adjusted quarterly series. The estimated seasonally adjusted 
series is found to be almost indistinguishable from that obtained by seasonally adjusting the underlying quarterly data. The outline of this paper is as follows. In Section 2 we show how to derive seasonally adjusted quarterly growth rates from the quarterly-reported annual growth rates. Section 3 presents the empirical illustration. Section 4 concludes.

\section{Theoretical background}

Consider the (unobserved) series of quarterly values for a variable, $y_{t}, t=1, \ldots, T$. Equivalently, we could consider the series of (unobserved) quarterly growth rates, $g_{t}^{Q}, t=2, \ldots, T$, where $g_{t}^{Q}=y_{t}-y_{t-1}$. Suppose that instead of $y_{t}$ or $g_{t}^{Q}$, we observe only the quarterly series of annual growth rates, $g_{t}^{A}, t=5, \ldots, T$, where $g_{t}^{A}=y_{t}-y_{t-4}$. The underlying quarterly growth series, $g_{t}^{Q}$, cannot be retrieved from the annual growth series, $g_{t}^{A}$, because there are $T-1$ observations on $g_{t}^{Q}$ but only $T-4$ observations on $g_{t}^{A}$. However, if we set $y_{1}=100$ and arbitrarily choose values for $g_{2}^{Q}, g_{3}^{Q}$ and $g_{4}^{Q}$, denoted $\hat{g}_{2}^{Q}, \hat{g}_{3}^{Q}$ and $\hat{g}_{4}^{Q}$, we can generate an arbitrary index series, $\hat{y}_{t}$, that, by construction, satisfies $g_{t}^{A}, t=5, \ldots, T$ :

$$
\begin{aligned}
& \hat{y}_{1}=100 \\
& \hat{y}_{2}=\hat{y}_{1}+\hat{g}_{2}^{Q} \\
& \hat{y}_{3}=\hat{y}_{2}+\hat{g}_{3}^{Q} \\
& \hat{y}_{4}=\hat{y}_{3}+\hat{g}_{4}^{Q} \\
& \hat{y}_{5}=\hat{y}_{1}+g_{5}^{A} \\
& \hat{y}_{6}=\hat{y}_{2}+g_{6}^{A} \\
& \vdots \\
& \hat{y}_{T}=\hat{y}_{T-4}+g_{T}^{A}
\end{aligned}
$$

\footnotetext{
${ }^{1}$ For simplicity, the analysis is conducted in terms of natural logarithms, with growth rates representing logarithmic changes. Clearly, it is straightforward to apply the results to data that is reported in non-logarithmic form by simply first transforming the data. Note also that while the analysis is conducted in terms of quarterly data, exactly the same procedure could be applied to data recorded at other frequencies.
} 
From this series, we can compute the corresponding quarterly growth rate series, $\hat{g}_{t}^{Q}=\hat{y}_{t}-\hat{y}_{t-1}, t=2, \ldots, T$. We can write $\hat{g}_{t}^{Q}$ for each quarter in terms of the three initial arbitrary quarterly growth rates, $\hat{g}_{2}^{Q}, \hat{g}_{3}^{Q}$ and $\hat{g}_{4}^{Q}$, and the observed annual growth rates, $g_{t}^{A}$, as follows.

$$
\begin{aligned}
& \text { Q1: } \hat{g}_{t}^{Q}=\hat{y}_{1}-\hat{y}_{4}+\sum_{s=0}^{(t-5) / 4}\left(g_{t-4 s}^{A}-g_{t-4 s-1}^{A}\right)+g_{4}^{A} \\
& \text { Q2: } \hat{g}_{t}^{Q}=\hat{y}_{2}-\hat{y}_{1}+\sum_{s=0}^{(t-6) / 4}\left(g_{t-4 s}^{A}-g_{t-4 s-1}^{A}\right) \\
& \text { Q3: } \hat{g}_{t}^{Q}=\hat{y}_{3}-\hat{y}_{2}+\sum_{s=0}^{(t-7) / 4}\left(g_{t-4 s}^{A}-g_{t-4 s-1}^{A}\right) \\
& \text { Q4: } \hat{g}_{t}^{Q}=\hat{y}_{4}-\hat{y}_{3}+\sum_{s=0}^{(t-8) / 4}\left(g_{t-4 s}^{A}-g_{t-4 s-1}^{A}\right)
\end{aligned}
$$

Noting that $g_{t}^{A}-g_{t-1}^{A}=g_{t}^{Q}-g_{t-4}^{Q}$ and that $g_{5}^{A}=g_{2}^{Q}+g_{3}^{Q}+g_{4}^{Q}+g_{5}^{Q}$, we can write these as

$$
\begin{aligned}
& \text { Q1: } \hat{g}_{t}^{Q}=g_{t}^{Q}+g_{2}^{Q}+g_{3}^{Q}+g_{4}^{Q}-\hat{g}_{2}^{Q}-\hat{g}_{3}^{Q}-\hat{g}_{4}^{Q} \\
& \text { Q2: } \hat{g}_{t}^{Q}=g_{t}^{Q}+\hat{g}_{2}^{Q}-g_{2}^{Q} \\
& \text { Q3: } \hat{g}_{t}^{Q}=g_{t}^{Q}+\hat{g}_{3}^{Q}-g_{3}^{Q} \\
& \text { Q4: } \hat{g}_{t}^{Q}=g_{t}^{Q}+\hat{g}_{4}^{Q}-g_{4}^{Q}
\end{aligned}
$$

Or, more succinctly, as

$$
\hat{g}_{t}^{Q}=g_{t}^{Q}+\sum_{i=1}^{4} d_{i, t} \hat{x}_{i}, \quad t=2, \ldots, T
$$

where $\quad \hat{x}_{1}=g_{2}^{Q}+g_{3}^{Q}+g_{4}^{Q}-\hat{g}_{2}^{Q}-\hat{g}_{3}^{Q}-\hat{g}_{4}^{Q}, \quad \hat{x}_{2}=\hat{g}_{2}^{Q}-g_{2}^{Q}, \quad \hat{x}_{3}=\hat{g}_{3}^{Q}-g_{3}^{Q}$, $\hat{x}_{4}=\hat{g}_{4}^{Q}-g_{4}^{Q}$ and $d_{i t}, i=1, \ldots, 4$, is a dummy variable that is equal to 1 in quarter $i$ and zero otherwise. Thus the arbitrary quarterly growth rate series, $\hat{g}_{t}^{Q}$, differs from 
the true quarterly growth rate series, $g_{t}^{Q}$, by a fixed additive seasonal, $\hat{x}_{i}, i=1, \ldots, 4$. Clearly, it is not possible to determine the 'correct' values of $\hat{g}_{2}^{Q}, \hat{g}_{3}^{Q}$ and $\hat{g}_{4}^{Q}$ that generate the underlying series, $g_{t}^{Q}$. However, it is possible to retrieve the seasonally adjusted series that is given by

$$
\widetilde{g}_{t}^{Q}=g_{t}^{Q}+\bar{g}^{Q}-\sum_{i=1}^{4} \alpha_{i} d_{i, t}, \quad t=2, \ldots, T
$$

where $\alpha_{i}=\operatorname{cov}\left(g_{t}^{Q}, d_{i, t}\right) / \operatorname{var}\left(d_{i, t}\right), i=1, \ldots, 4$, are the population slope coefficients in a regression of the true quarterly growth series, $g_{t}^{Q}$, on $d_{i t}, i=1, \ldots, 4$, and $\bar{g}^{Q}$ is the mean of $g_{t}^{Q}$. This is formalised in the following proposition

Proposition 1: The seasonally adjusted quarterly growth series can be computed as

$$
\widetilde{g}_{t}^{Q}=\hat{g}_{t}^{Q}+\overline{\hat{g}}^{Q}-\sum_{i=1}^{4} \hat{\alpha}_{i} d_{i, t}, \quad t=2, \ldots, T
$$

where $\hat{\alpha}_{i}=\operatorname{cov}\left(\hat{g}_{t}^{Q}, d_{i, t}\right) / \operatorname{var}\left(d_{i, t}\right), i=1, \ldots, 4$, are the population slope coefficients in a regression of the arbitrary quarterly growth series, $\hat{g}_{t}^{Q}$, on $d_{i t}, i=1, \ldots, 4$, and $\overline{\hat{g}}^{Q}$ is the mean of $\hat{g}_{t}^{Q}$.

Proof: See Appendix.

Thus, while the true unadjusted series, $g_{t}^{Q}$, is unobservable, the true dummy-variable seasonally adjusted series, $\widetilde{g}_{t}^{Q}$, can be obtained by seasonally adjusting the arbitrary series, $\hat{g}_{t}^{Q}$. Intuitively, this is because the arbitrary series, $\hat{g}_{t}^{Q}$, differs from the underlying series, $g_{t}^{Q}$, by a fixed seasonal, $\hat{x}_{i}$, while the true seasonally adjusted series, $\widetilde{g}_{t}^{Q}$, differs from the underlying series, $g_{t}^{Q}$, by a fixed seasonal, $\tilde{x}_{i}$. Thus $\hat{g}_{t}^{Q}$ and $\tilde{g}_{t}^{Q}$ differ by the fixed seasonal $\hat{x}_{i}-\tilde{x}_{i}$. Seasonally adjusting $\hat{g}_{t}^{Q}$ removes this seasonal to yield $\widetilde{g}_{t}^{Q}$. From the seasonally adjusted quarterly growth series, we can 
trivially compute the corresponding seasonally adjusted levels series, $\tilde{y}_{t}, t=1, \ldots, T$, in index form. Since empirical work in economics typically employs seasonally adjusted data, this should be sufficient for most studies that require quarterly observations on an economic series. Note that an interesting corollary to Proposition 1 is that if the underlying data does not display quarterly seasonality, then this procedure will retrieve the underlying quarterly series itself. To the extent that many economic series display little seasonality, the procedure is likely to generate an accurate approximation to the underlying quarterly data.

Noting from equation (10) that we only require the seasonally adjusted values of $g_{2}^{Q}$, $g_{3}^{Q}$ and $g_{4}^{Q}$ in order to generate the entire seasonally adjusted series, an alternative way of computing the seasonally adjusted series, $\widetilde{g}_{t}^{Q}$, is given by the following proposition.

Proposition 2: If $\hat{g}_{2}^{Q}, \hat{g}_{3}^{Q}$ and $\hat{g}_{4}^{Q}$ are chosen in order to minimise the variance of $\hat{g}_{t}^{Q}, t=5, \ldots, T$, then the resulting series of estimated quarterly growth rates, $\hat{g}_{t}^{Q}$, $t=2, \ldots, T$, will be equal to the seasonally adjusted quarterly growth rates, $\widetilde{g}_{t}^{Q}$, $t=2, \ldots, T$.

Proof: See Appendix.

While yielding exactly the same result as Proposition 1, the approach of Proposition 2 may be computationally simpler. In particular, the variance of $\hat{g}_{t}^{Q}$ can be easily minimised using a numerical routine such as Excel's Solver function.

\section{More Sophisticated Seasonal Adjustment Methods}

The seasonal adjustment method considered above is rudimentary in that it simply removes a fixed additive seasonal from the true quarterly growth series. In practice, it is common to use more sophisticated seasonal adjustment methods that include empirical-based approaches, such as the Bureau of Census X11 method (see Shiskin et al, 1967), and model-based approaches that employ signal extraction techniques 
(see, for example, Burridge and Wallis, 1984). Consider the general seasonal adjustment procedure $\widetilde{g}_{t}^{Q}=S A\left(g_{t}^{Q}\right)$. If we assume that the seasonal adjustment procedure is linearly separable in terms of the additive seasonal dummies, and that the seasonally adjusted values of the additive seasonal dummies are zero then clearly we can use the arbitrary series, $\hat{g}_{t}^{Q}$, in place of the true series, $g_{t}^{Q}$, in the seasonal adjustment procedure in order to obtain the true seasonally adjusted series, $\widetilde{g}_{t}^{Q}$, since we have

$$
\begin{aligned}
\widetilde{g}_{t}^{Q} & =S A\left(g_{t}^{Q}\right) \\
& =S A\left(\hat{g}_{t}^{Q}-\sum_{i=1}^{4} d_{i, t} \hat{x}_{i}\right) \\
& =S A\left(\hat{g}_{t}^{Q}\right)-S A\left(\sum_{i=1}^{4} d_{i, t} \hat{x}_{i}\right) \\
& =S A\left(\hat{g}_{t}^{Q}\right)
\end{aligned}
$$

This class of seasonal adjustment methods would include those that are consistent with the Holt-Winters additive decomposition of a time series (see Holt et al, 1960; Winters, 1960), or its equivalent ARMA representation (see, for example, Harvey, 1984). To the extent that some seasonal adjustment procedures are not linearly separable in terms of the additive seasonal effects (in particular, those that allow for the seasonal component to vary with the level of the series), using $\hat{g}_{t}^{Q}$ in place $g_{t}^{Q}$ will provide only an approximation to the true seasonally adjusted series. We investigate the accuracy of this approximation in the following section.

\section{Empirical illustration}

In this section we apply the above methodology to estimate the seasonally adjusted quarterly growth of the UK Retail Sales series, which has very significant quarterly seasonality. Quarterly non-seasonally adjusted data were obtained from Datastream for the period 30 June 1995 to 30 June 2005 (Datastream code UKRETTOTH). Quarterly growth rates were then calculated as the first difference of the natural logarithm of this series. Of course, for the UK Retail Sales series there is no need to employ the procedure described in the previous section since the underlying quarterly 
data are readily available and can be seasonally adjusted directly. However, the purpose of this section is to demonstrate the accuracy of our procedure relative to more sophisticated seasonal adjustment methods such as those used by commercial data providers such as Datastream, and for that, we require the underlying quarterly growth data. To provide a benchmark for our procedure, the data were seasonally adjusted in two ways. Firstly, the actual quarterly growth data were regressed on quarterly dummy variables and the seasonally adjusted series constructed using equation (10). This is termed the DV-adjusted series. Secondly, the data were seasonally adjusted using the ratio-to-moving-average approach that is employed by Datastream. This procedure first takes the ratio of the data to a centred one-year moving average each quarter, excluding the first two and last two quarters, and then uses the five-year centred moving average of this ratio to adjust the original data. The first two and last two quarters of the data are adjusted by the adjacent five-year moving averages. Finally, the resulting observations are adjusted by a factor each year to ensure that the annual sum of the seasonally adjusted data is the same as the annual sum of the original data. ${ }^{2}$ This series is termed the RTMA-adjusted series. Figure 1 plots the quarterly logarithmic growth series for UK Retail Sales. There is clear evidence of significant quarterly seasonality with sales rising in Q4 and falling in Q1 of every year.

We applied the procedure described in the previous section to estimate both the DVadjusted series and the RTMA-adjusted series. We first computed annual growth rates for the Retail Sales series and used these to construct an arbitrary quarterly logarithmic index series, $\hat{y}_{t}$, using equation (1) with $\hat{g}_{2}^{Q}=\hat{g}_{3}^{Q}=\hat{g}_{4}^{Q}=0$. This was then used to compute the corresponding (arbitrary) quarterly growth series, $\hat{g}_{t}^{Q}=\hat{y}_{t}-\hat{y}_{t-1}$. To retrieve the DV-adjusted series, $\hat{g}_{t}^{Q}$ was regressed on quarterly dummy variables and the seasonally adjusted series constructed using equation (11). To estimate the RTMA-adjusted series, we seasonally adjusted the arbitrary quarterly series, $\hat{y}_{t}$, using Datastream's ratio-to-moving-average seasonal adjustment procedure, and then computed the quarterly growth rates of the resulting series. Figure

\footnotetext{
${ }^{2}$ In order to apply this seasonal adjustment procedure, the actual quarterly series was saved in Datastream as a User Defined Time Series and then retrieved as a seasonally adjusted series.
} 
2 plots the actual DV-adjusted series and the estimated DV-adjusted series. As expected from Proposition 1 of the previous section, the two are identical. Figure 3 plots the actual RTMA-adjusted series and the estimated RTMA-adjusted series. While not identical, the two series are almost indistinguishable, with only very minor discrepancies occurring at some of the turning points. Indeed, the correlation coefficient between these two series is 0.992. This confirms that the procedure described in the previous section can be used to retrieve not only the rudimentary dummy-variable seasonally adjusted series, but also series that are seasonally adjusted using more sophisticated methods.

\section{Conclusion}

For many economic series, official statistical agencies report annual (or 'year-onyear') growth rates rather than the underlying quarterly data themselves. Since a given quarterly series of annual growth rates is consistent with an infinite number of possible series of quarterly growth rates, it is clearly not possible to retrieve the underlying (unadjusted) quarterly data from the observed annual growth rates. In this paper, however, we show that while the original data themselves cannot be identified, we can retrieve the seasonally adjusted quarterly growth rates and from these, impute the seasonally adjusted series itself, in index form. As an illustration, we apply our technique to the UK Retail Sales series, retrieving the true dummy-variable seasonally adjusted series exactly, and the ratio-to-moving average seasonally adjusted series with a very high degree of accuracy. The procedure outlined in this paper will undoubtedly be useful for both practitioners and academics. 


\section{Appendix}

Proof of Proposition 1: Noting that $\bar{g}^{Q}=\frac{1}{4} \sum_{i=1}^{4} \alpha_{i}$, the true seasonally adjusted quarterly growth series given by (11) can be written as

$$
\widetilde{g}_{t}^{Q}=g_{t}^{Q}+\frac{1}{4} \sum_{i=1}^{4} \alpha_{i}-\sum_{i=1}^{4} \alpha_{i} d_{i, t}
$$

Consider the seasonally adjusted series

$$
\tilde{\hat{g}}_{t}^{Q}=\hat{g}_{t}^{Q}+\frac{1}{4} \sum_{i=1}^{4} \hat{\alpha}_{i}-\sum_{i=1}^{4} \hat{\alpha}_{i} d_{i, t}
$$

Using equation (10) and noting that $\operatorname{cov}\left(d_{i, t}, d_{j, t}\right)=0$ for $i \neq j$, we can write the slope coefficients as

$$
\begin{aligned}
\hat{\alpha}_{i} & =\operatorname{cov}\left(g_{t}^{Q}+\sum_{i=1}^{4} d_{i, t} \hat{x}_{i}\right) / \operatorname{var}\left(d_{i, t}\right) \\
& =\operatorname{cov}\left(g_{t}^{Q}, d_{i, t}\right) / \operatorname{var}\left(d_{i, t}\right)+\operatorname{cov}\left(\sum_{i=1}^{4} d_{i, t} \hat{x}_{i}\right) / \operatorname{var}\left(d_{i, t}\right) \\
& =\operatorname{cov}\left(g_{t}^{Q}, d_{i, t}\right) / \operatorname{var}\left(d_{i, t}\right)+\operatorname{cov}\left(d_{i, t} \hat{x}_{i}, d_{i, t}\right) / \operatorname{var}\left(d_{i, t}\right) \\
& =\alpha_{i}+\hat{x}_{i}
\end{aligned}
$$

Substituting into (A2), we have

$$
\begin{aligned}
\tilde{\hat{g}}_{t}^{Q} & =\hat{g}_{t}^{Q}+\frac{1}{4} \sum_{i=1}^{4}\left(\alpha_{i}+\hat{x}_{i}\right)-\sum_{i=1}^{4}\left(\alpha_{i}+\hat{x}_{i}\right) d_{i, t} \\
& =g_{t}^{Q}+\sum_{i=1}^{4} d_{i, t} \hat{x}_{i}+\frac{1}{4} \sum_{i=1}^{4}\left(\alpha_{i}+\hat{x}_{i}\right)-\sum_{i=1}^{4}\left(\alpha_{i}+\hat{x}_{i}\right) d_{i, t} \\
& =g_{t}^{Q}+\frac{1}{4} \sum_{i=1}^{4}\left(\alpha_{i}+\hat{x}_{i}\right)-\sum_{i=1}^{4} \alpha_{i} d_{i, t} \\
& =g_{t}^{Q}+\frac{1}{4} \sum_{i=1}^{4} \alpha_{i}-\sum_{i=1}^{4} \alpha_{i} d_{i, t}
\end{aligned}
$$

with the last line following since $\sum_{i=1}^{4} \hat{x}_{i}=0$. This is the same as the true seasonally adjusted quarterly growth given by equation (A1). 
Proof of Proposition 2: We can rewrite the arbitrary quarterly growth series, $\hat{g}_{t}^{Q}$, given by equation (10) as

$$
\hat{g}_{t}^{Q}=g_{t}^{Q}+\sum_{i=2}^{4} \delta_{i, t}\left(\hat{g}_{i}^{Q}-g_{i}^{Q}\right)
$$

where $\delta_{i, t}=d_{i, t}-d_{1, t}, i=2, \ldots, 4$. Taking the variance of (A5) and noting that $\operatorname{var}\left(\delta_{i, t}\right)=1 / 2$ and $\operatorname{cov}\left(\delta_{i, t}, \delta_{j, t}\right)=1 / 4$ for $i \neq j$, we have

$$
\begin{aligned}
\operatorname{var}\left(\hat{g}_{t}^{Q}\right) & =\operatorname{var}\left(g_{t}^{Q}\right)+(1 / 2)\left(\hat{g}_{2}^{Q}-g_{2}^{Q}\right)^{2}+(1 / 2)\left(\hat{g}_{3}^{Q}-g_{3}^{Q}\right)^{2}+(1 / 2)\left(\hat{g}_{4}^{Q}-g_{4}^{Q}\right)^{2} \\
& +2 \operatorname{cov}\left(g_{t}^{Q}, \delta_{2, t}\right)\left(\hat{g}_{2}^{Q}-g_{2}^{Q}\right)+2 \operatorname{cov}\left(g_{t}^{Q}, \delta_{3, t}\right)\left(\hat{g}_{3}^{Q}-g_{3}^{Q}\right) \\
& +2 \operatorname{cov}\left(g_{t}^{Q}, \delta_{4, t}\right)\left(\hat{g}_{4}^{Q}-g_{4}^{Q}\right) \\
& +(1 / 2)\left(\hat{g}_{2}^{Q}-g_{2}^{Q}\right)\left(\hat{g}_{2}^{Q}-g_{2}^{Q}\right)+(1 / 2)\left(\hat{g}_{2}^{Q}-g_{2}^{Q}\right)\left(\hat{g}_{4}^{Q}-g_{4}^{Q}\right) \\
& +(1 / 2)\left(\hat{g}_{3}^{Q}-g_{3}^{Q}\right)\left(\hat{g}_{4}^{Q}-g_{4}^{Q}\right)
\end{aligned}
$$

Differentiating with respect to $\hat{g}_{2}^{Q}, \hat{g}_{3}^{Q}$ and $\hat{g}_{4}^{Q}$ and setting to zero yields the following first order conditions.

$$
\begin{aligned}
& \frac{\partial \operatorname{var}\left(\hat{g}_{t}^{Q}\right)}{\partial \hat{g}_{2}^{Q}}=\hat{g}_{2}^{Q}-g_{2}^{Q}+2 \operatorname{cov}\left(g_{t}^{Q}, \delta_{2, t}\right)+\frac{1}{2}\left(\hat{g}_{3}^{Q}-g_{3}^{Q}\right)+\frac{1}{2}\left(\hat{g}_{4}^{Q}-g_{4}^{Q}\right)=0 \\
& \frac{\partial \operatorname{var}\left(\hat{g}_{t}^{Q}\right)}{\partial \hat{g}_{3}^{Q}}=\hat{g}_{3}^{Q}-g_{3}^{Q}+2 \operatorname{cov}\left(g_{t}^{Q}, \delta_{3, t}\right)+\frac{1}{2}\left(\hat{g}_{2}^{Q}-g_{2}^{Q}\right)+\frac{1}{2}\left(\hat{g}_{4}^{Q}-g_{4}^{Q}\right)=0 \\
& \frac{\partial \operatorname{var}\left(\hat{g}_{t}^{Q}\right)}{\partial \hat{g}_{4}^{Q}}=\hat{g}_{4}^{Q}-g_{4}^{Q}+2 \operatorname{cov}\left(g_{t}^{Q}, \delta_{4, t}\right)+\frac{1}{2}\left(\hat{g}_{2}^{Q}-g_{2}^{Q}\right)+\frac{1}{2}\left(\hat{g}_{3}^{Q}-g_{3}^{Q}\right)=0
\end{aligned}
$$

Solving for $\hat{g}_{2}^{Q}, \hat{g}_{3}^{Q}$ and $\hat{g}_{4}^{Q}$ yields

$$
\begin{aligned}
& \hat{g}_{2}^{Q}=g_{2}^{Q}-3 \operatorname{cov}\left(g_{t}^{Q}, \delta_{2, t}\right)+\operatorname{cov}\left(g_{t}^{Q}, \delta_{3, t}\right)+\operatorname{cov}\left(g_{t}^{Q}, \delta_{4, t}\right) \\
& \hat{g}_{3}^{Q}=g_{3}^{Q}-3 \operatorname{cov}\left(g_{t}^{Q}, \delta_{3, t}\right)+\operatorname{cov}\left(g_{t}^{Q}, \delta_{2, t}\right)+\operatorname{cov}\left(g_{t}^{Q}, \delta_{4, t}\right) \\
& \hat{g}_{4}^{Q}=g_{4}^{Q}-3 \operatorname{cov}\left(g_{t}^{Q}, \delta_{4, t}\right)+\operatorname{cov}\left(g_{t}^{Q}, \delta_{2, t}\right)+\operatorname{cov}\left(g_{t}^{Q}, \delta_{3, t}\right)
\end{aligned}
$$

Substituting into equations (6) to (9) in the main text yields the following expressions for the estimated quarterly growth rate in each period

$$
\begin{aligned}
& \text { Q1: } \hat{g}_{t}^{Q}=g_{t}^{Q}-3 \operatorname{cov}\left(g_{t}^{Q}, d_{1, t}\right)+\operatorname{cov}\left(g_{t}^{Q}, d_{2, t}\right)+\operatorname{cov}\left(g_{t}^{Q}, d_{3, t}\right)+\operatorname{cov}\left(g_{t}^{Q}, d_{4, t}\right) \\
& \text { Q2: } \hat{g}_{t}^{Q}=g_{t}^{Q}-3 \operatorname{cov}\left(g_{t}^{Q}, d_{2, t}\right)+\operatorname{cov}\left(g_{t}^{Q}, d_{1, t}\right)+\operatorname{cov}\left(g_{t}^{Q}, d_{3, t}\right)+\operatorname{cov}\left(g_{t}^{Q}, d_{4, t}\right) \\
& \text { Q3: } \hat{g}_{t}^{Q}=g_{t}^{Q}-3 \operatorname{cov}\left(g_{t}^{Q}, d_{3, t}\right)+\operatorname{cov}\left(g_{t}^{Q}, d_{1, t}\right)+\operatorname{cov}\left(g_{t}^{Q}, d_{2, t}\right)+\operatorname{cov}\left(g_{t}^{Q}, d_{4, t}\right) \\
& \text { Q4: } \hat{g}_{t}^{Q}=g_{t}^{Q}-3 \operatorname{cov}\left(g_{t}^{Q}, d_{4, t}\right)+\operatorname{cov}\left(g_{t}^{Q}, d_{1, t}\right)+\operatorname{cov}\left(g_{t}^{Q}, d_{2, t}\right)+\operatorname{cov}\left(g_{t}^{Q}, d_{3, t}\right)
\end{aligned}
$$

Or, more succinctly,

$$
\hat{g}_{t}^{Q}=g_{t}^{Q}+\frac{1}{4} \sum_{i=1}^{4} \alpha_{i}-\sum_{i=1}^{4} \alpha_{i} d_{i, t}
$$

where $\alpha_{i}=\operatorname{cov}\left(g_{t}^{Q}, d_{i, t}\right)$. This is the seasonally adjusted quarterly growth rate given in the main text by equation (11). 


\section{References}

Burridge, P., and K. Wallis, 1984, "Unobserved-Components Models for Seasonal Adjustment Filters", Journal of Business and Economic Statistics 2, 350-359.

Harvey, A, 1984, “A Unified View of Statistical Forecasting Procedures", Journal of Forecasting 3, 245-275.

Holt, C., F. Modigliani, J. Muth and H. Simon, 1960, Planning Production Inventories and Work Force, Prentice-Hall.

Shishkin, J., A Young and J. Musgrave, 1967, "The X-11 Variant of the Census Method II Seasonal Adjustment Program", Technical Paper No. 15, US Department of Commerce, Bureau of Economic Analysis.

Winters, P., 1960, "Forecasting Sales by Exponentially Weighted Moving Averages", Management Science 6, 324-342. 
Figure 1: Quarterly Log Growth in UK Retail Sales

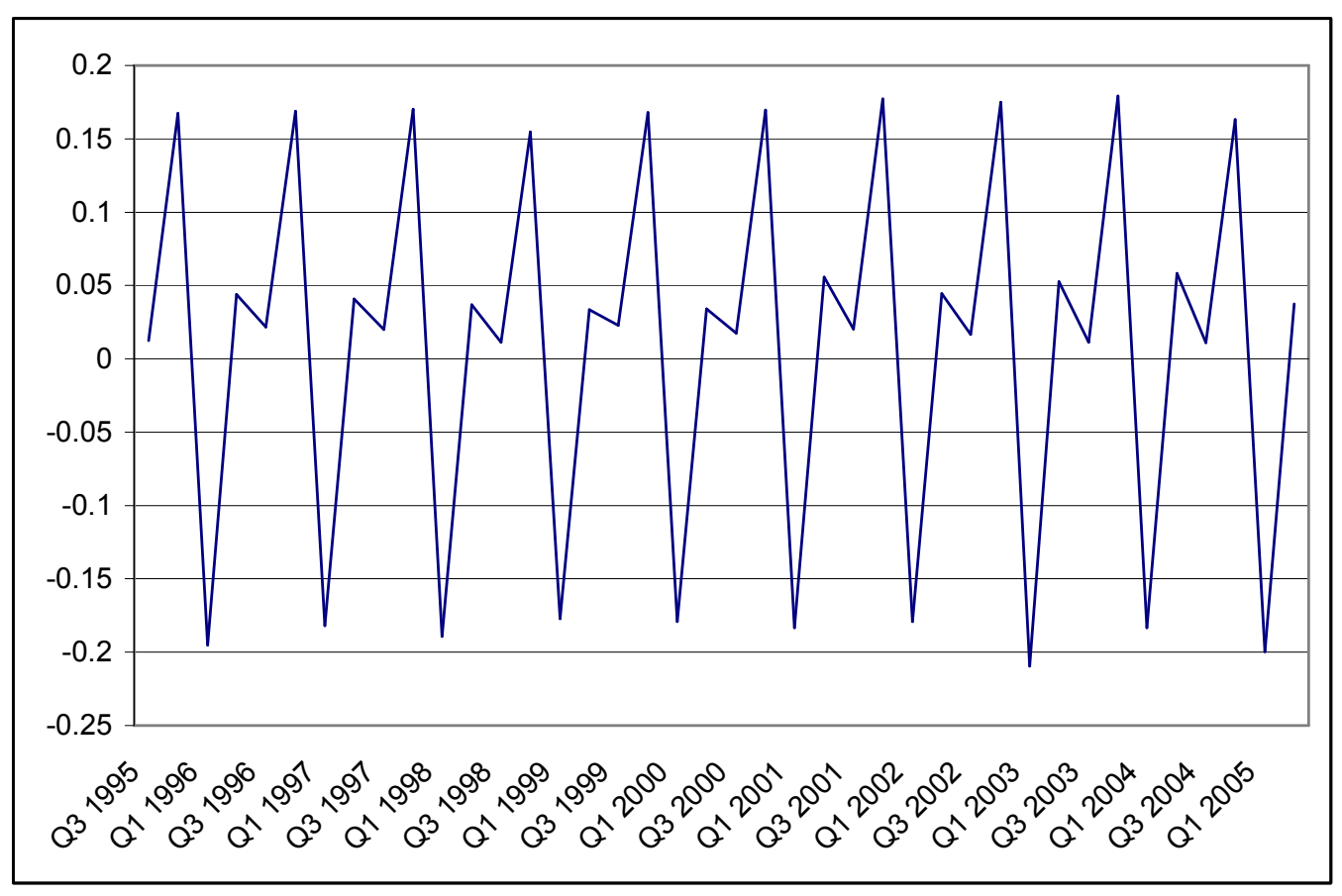


Figure 2: Dummy-Variable Seasonally Adjusted Quarterly Log Growth in UK Retail Sales

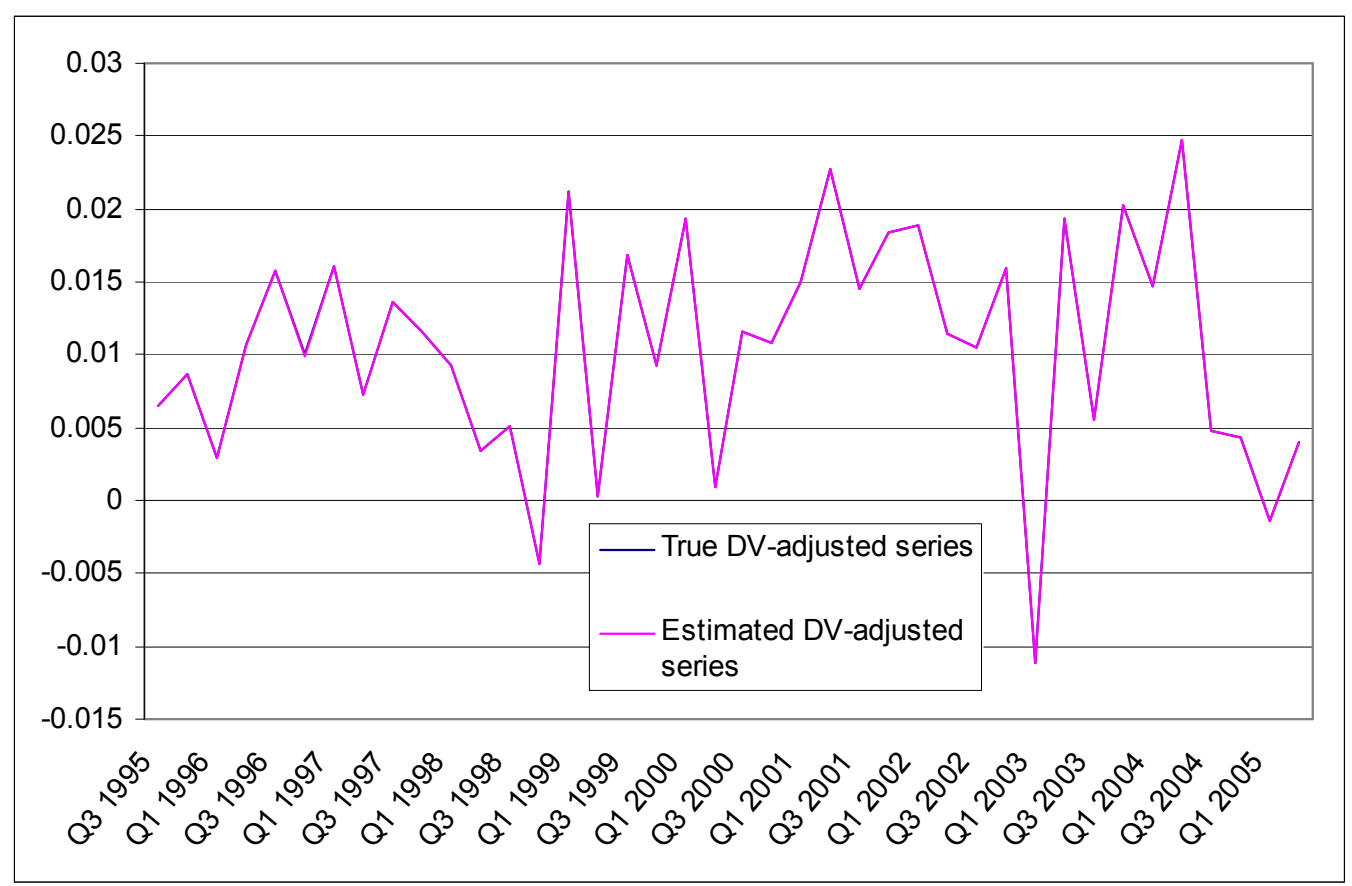


Figure 3: Ratio-to-Moving-Average Seasonally Adjusted Quarterly Log Growth in UK Retail Sales

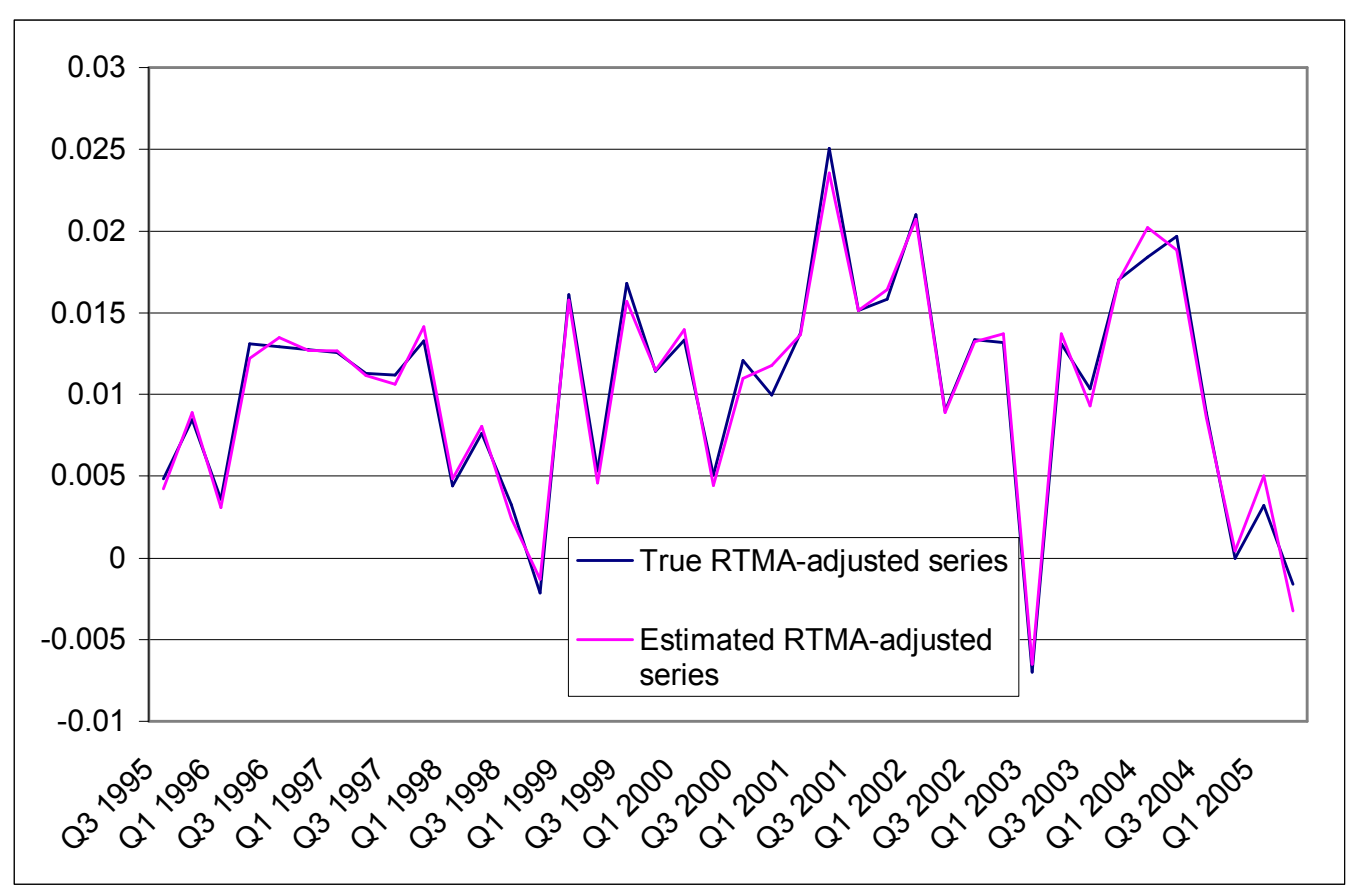

